\title{
ELECTRONIC SPECTRA OF SALICYLAMIDE IN POLYCRYSTALLINE FORM AND SOLID ENVIRONMENT*
}

\author{
M. KaKaš, I. JaNIĆ and I. GÚth \\ Institute of Physics, Faculty of Science, University of Novi Sad \\ Trg D: Obradovića 4, 21000 Novi Sad, Yugoslavia
}

(Received July 23, 1998; in final form December 16, 1998)

\begin{abstract}
The fluorescence emission of crystalline salicylamide $\left(\lambda_{\max }=426 \mathrm{~nm}\right.$, Stokes shift $6500 \mathrm{~cm}^{-1}$ ) is attributed to the proton-transferred species. We suppose that the peak at $359 \mathrm{~nm}$ in the substructure of fluorescence excitation spectrum at $77 \mathrm{~K}$ is in fact the position of the $0-0$ transition. The luminescence and excitation spectra of protolytic forms (anion, neutral molecule and cation) of salicylamide in solid solutions were also examined, as well as the equilibria and photoprocesses in the excited state.
\end{abstract}

PACS numbers: $33.50 . \mathrm{Dq}$

\section{Introduction}

Salicylamide (SAM), as other salicylic derivatives, belongs to a class of molecules forming strong intramolecular $\mathrm{H}$-bond in the ground electronic state. A characteristic feature of the emission of these molecules is the appearance of a fluorescence band with' a large Stokes shift. Depending on the solvent nature, temperature and excitation wavelength, there can appear one more emission band, fluorescent or phosphorescent, which is located at a shorter wavelength with respect to the first, main fluorescence band (see Fig. 2). Weller [1] was the first who explained the origin of the main fluorescence band in terms of the fast proton transfer along the intramolecular H-bond resulting in resonance-stabilized zwitterion. The origin of the shortwave emission band, which is usually of a considerably lower intensity, is generally attributed to the existence of the other conformer of the ground state tautomeric form of the molecule [2-5]. For SAM molecule, this is illustrated in Fig. 1, like in Ref. [6], where the aqueous solutions of SAM were examined. In the ground state, there exist the cis- (a) and trans-conformer (c) of

*The results of this paper were initially presented at The Jabtonski Centennial Conference on Luminescence and Photophysics, July 23-27, 1998, Toruń, Poland. 


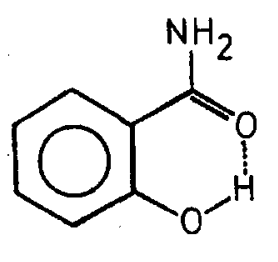

a

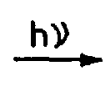

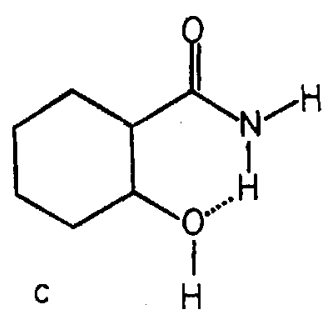

Fig. 1. Conformational forms of salicylamide.

the enol tautomer in which hydrogen atom is located at the hydroxy oxygen. Excitation of the cis-conformer leads to the intramolecular proton transfer along the $\mathrm{H}$-bond between two oxygens to give the excited zwitterion (b), in which the hydrogen atom is located at the carbonyl oxygen. Excitation of the trans-conformer (c), with intramolecular $\mathrm{H}$-bond between hydroxy oxygen and amide nitrogen, does not lead to proton transfer but gives an excited neutral enol form, responsible for the shortwave emission. An ab initio calculation, performed later [7], showed that the most stable conformer is the cis-form (a) and less stable (by $18.60 \mathrm{~kJ} / \mathrm{mol}$ ) is the trans-form (c). Examination of SAM by two-step laser excitation [8] and high-resolution fluorescence spectroscopy in mixed durene crystal [9], as well as recent theoretical consideration [10], indicate that the zwitterionic form has no counterpart in the ground state. This fact makes possible to use SAM as an active medium in proton-transfer laser [11].

In spite of extensive investigation, there is little information on SAM in solid media such as solid solution and crystalline state. An exception is the study in durene mixed crystal at $4.2 \mathrm{~K}$ [9]. In this work, the luminescence and excitation spectra of SAM, recorded for different solid solutions and polycrystalline form at both room temperature and $77 \mathrm{~K}$, were examined.

\section{Experimental}

Salicylamide was synthesized by the method described elsewhere [12]. The obtained product was recrystallized three times from water. The solvents used were of spectroscopic or analytical grade and their purity was checked spectroscopically. Aqueous solutions of different $\mathrm{pH}$ values were prepared as described earlier [13]. The concentrations of the solutions were $10^{-4}-10^{-3} \mathrm{~mol} / \mathrm{dm}^{3}$. The sample for recording spectra of polycrystalline SAM was a mixture of $\mathrm{MgO}$ powder and SAM crystal powder in a mass ratio of 100:1.

Diffusion-reflection spectrum was recorded at room temperature, using an SPM-2 quartz monochromator (Zeiss, Jena). The reflection cell was of R-45/0 type, and $\mathrm{MgO}$ was used as standard. The sample was prepared to comply with the Kubelka-Munk theory [14]. The absorption coefficient was analyzed by the reemission function $F$, based on the empirical Kubelka-Munk theory. The luminescence and excitation spectra were recorded on an Aminco-Bowman spectrophotofluorometer with an off-axis ellipsoidal mirror condensing system. The square quartz 
cuvette was used for liquid solutions at room temperature, and corresponding spectra were corrected for spectral response of equipment. The round quartz cuvette $(\phi=1 \mathrm{~mm})$ was used for polycrystalline samples at both room temperature and $77 \mathrm{~K}$, as well as for obtaining solid solutions, whereas liquid solution was placed in a micro Dewar flask with liquid nitrogen. Frozen aqueous solutions were made in a specially constructed cuvette. The presented spectra are uncorrected.

\section{Results and discussion}

In nonpolar aprotic solvents, SAM exhibits only one fluorescence band, with a large Stokes shift, at both room temperature and $77 \mathrm{~K}$. This band we attributed to the zwitterionic emission. Dual fluorescence of SAM in aqueous solutions of medium acidity at room temperature was obtained previously [6], as well as in our experiments. However, the intensity of shortwave band was very low compared to the longwave one, therefore it was difficult to determine its position. In ethanolic solution at room temperature, we registered dual fluorescence with a considerably higher relative intensity of the shortwave band. The positions of the bands maxima in the corrected spectrum were 343 and $434 \mathrm{~nm}$, while their intensity ratio was 1:6. The luminescence and excitation spectra of this solution at $77 \mathrm{~K}$ are presented in Fig. 2. The maxima of the fluorescence bands are at 334 and $427 \mathrm{~nm}$. The excitation

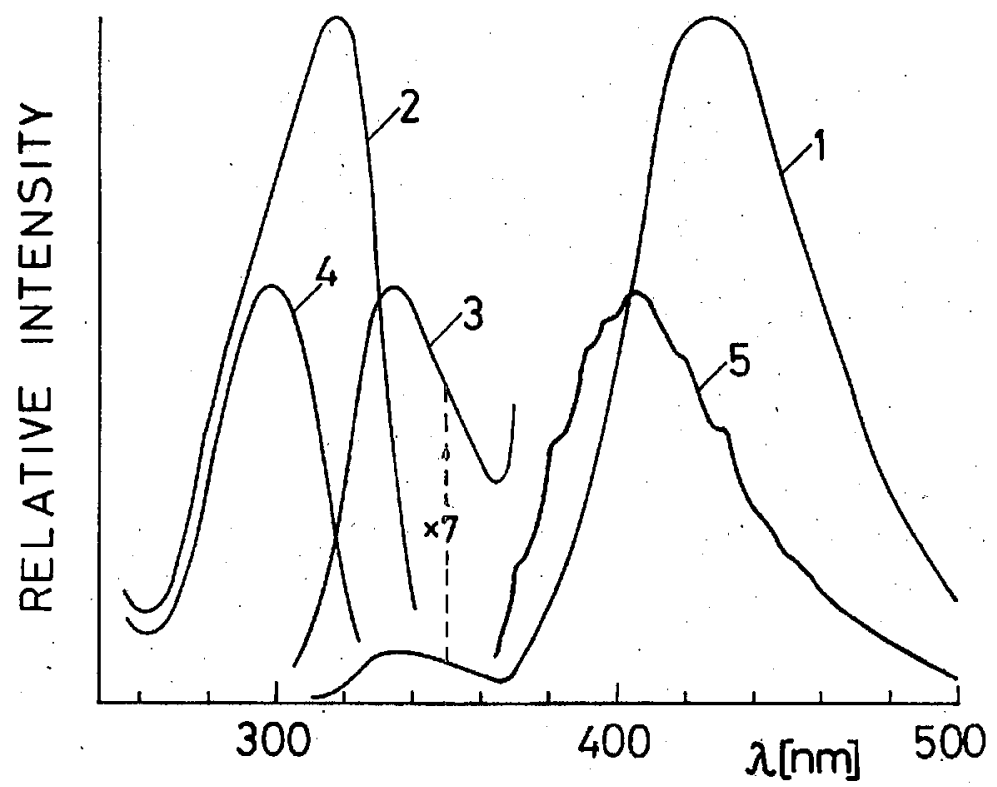

Fig. 2. Electronic spectra of salicylamide in ethanolic solution at $77 \mathrm{~K}: 1$ - dual fluorescence emission ( 334 and $427 \mathrm{~nm}$ ) with excitation at $280 \mathrm{~nm} ; 3$ - shortwave fluorescence (7-fold amplification); 2 - and 4-fluorescence excitation spectra obtained by monitoring at 460 and $334 \mathrm{~nm}$, respectively; 5 - phosphorescence emission spectrum obtained by excitation at $285 \mathrm{~nm}$. Concentration was $10^{-3} \mathrm{~mol} / \mathrm{dm}^{3}$. 
with a maximum at $298 \mathrm{~nm}$ (the same as at $293 \mathrm{~K}$ ) was obtained by monitoring the fluorescence at $334 \mathrm{~nm}$, while the excitation with a maximum at $318 \mathrm{~nm}(319 \mathrm{~nm}$ at $293 \mathrm{~K}$ ) was obtained by monitoring the fluorescence at $460 \mathrm{~nm}$. In addition to fluorescence, the phosphorescence with a maximum at $406 \mathrm{~nm}$ and vibrational substructure was also registered at $77 \mathrm{~K}$ (Fig. 2, curve 5). The phosphorescence excitation is the same as that obtained by monitoring fluorescence at $334 \mathrm{~nm}$. It is obvious that there are two emission species. The longwave fluorescence is due to the zwitterionic emission resulting in excitation of the cis-conformer, while the shortwave fluorescence and the corresponding phosphorescence is attributed to the emission of excited trans-conformer, stabilized by intermolecular H-bonds with solvent molecules.

In aqueous buffer solutions of appropriate $\mathrm{pH}$, apart from the neutral molecule it was also possible to register the anion, arising from the hydroxy group, and the cation, arising from protonation of the amide nitrogen. The corresponding equilibrium constants for these protolytic reactions are $\mathrm{pK}_{1}=8.89$, obtained previously [15], and $\mathrm{pK}_{2}=-2.32$, obtained in the present work. By the Förster cycle and the above equilibrium constants in the ground state, the corresponding constants in the excited state were calculated, and they are $\mathrm{pK}_{1}^{*}=2.5$ and $\mathrm{pK}_{2}^{*}=1.5$, respectively. The protolytic reaction in the excited state and partly established

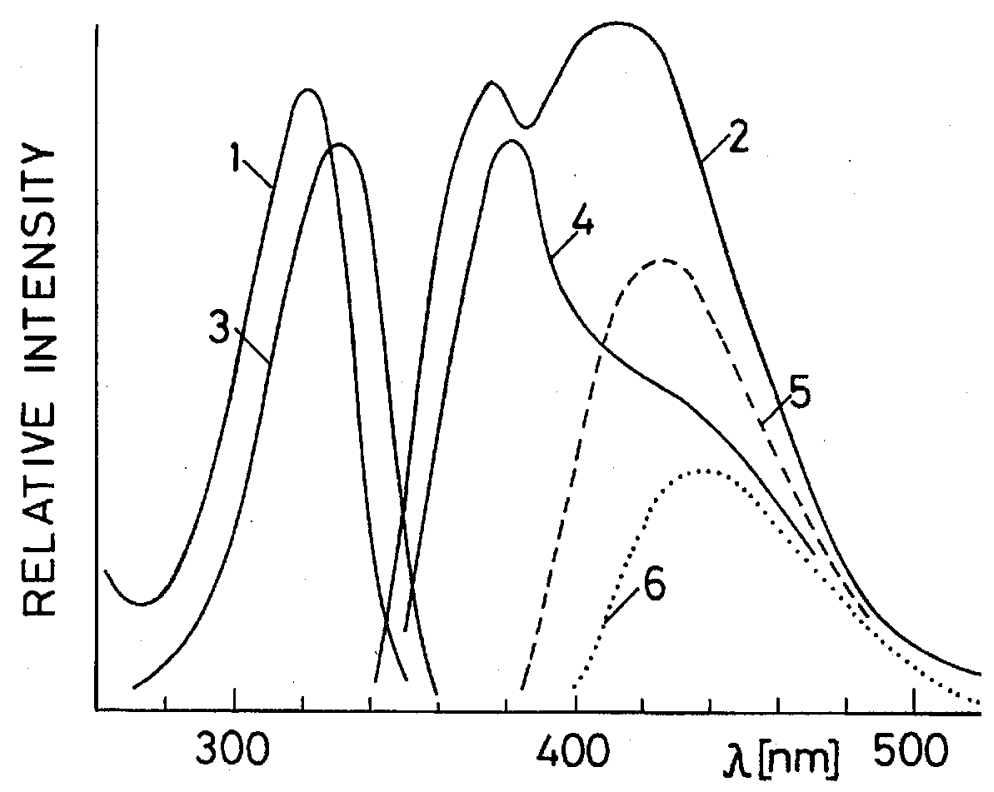

Fig. 3. Luminescence excitation ( 1 and 3 ), luminescence emission (2 and 4 ) and phosphorescence ( 5 and 6 ) spectra of salicylamide in aqueous solutions at $77 \mathrm{~K}: 3,4$ and $6-$ spectra of anion $(\mathrm{pH}=11.98) ; 1,2$ and $5-$ spectra of cation $\left(\mathrm{H}_{0}=-4.4\right)$. Concentration $10^{-4} \mathrm{~mol} / \mathrm{dm}^{3}$. 
equilibria make it difficult to register the molecular spectrum. Because of that, we examined in detail the spectra of the anion and cation in solid solutions at $77 \mathrm{~K}$, which are presented in Fig. 3. Both species exhibit the fluorescence as well as phosphorescence. The anion fluorescence and phosphorescence maxima are at 381 and $440 \mathrm{~nm}$, respectively, while those for cation are observed at 375 and $424 \mathrm{~nm}$, respectively. It is important to point out that the luminescence of the cation at $77 \mathrm{~K}$ appears from $\mathrm{pH}=1.8$ to lower values, and that of the anion (but not as the unique emission) from $\mathrm{pH}=1.8$ to higher values, which agrees with obtained equilibrium constants in the excited state. The maxima of the luminescence excitation spectra of the anion and cation are located at 331 and $321 \mathrm{~nm}$, respectively.

The spectra of polycrystalline SAM are shown in Fig. 4. Curve 1 represents the absorption of SAM crystal powder at room temperature, obtained by diffusion-reflection spectroscopy, with the maximum intensity at $326 \mathrm{~nm}$. The shift of this absorption to the longer wavelength compared to the absorption in liquid hydrocarbon solvent $\left(\lambda_{\max }=308 \mathrm{~nm}\right)$ is significant, which is the result of the strong influence of the crystal environment on the molecular structure, and thus on its energy. The fluorescence emission of polycrystalline SAM at room temperature $\left(\lambda_{\max }=426 \mathrm{~nm}\right)$ is located in the region of the longwave fluorescence band in solutions, so it is attributed to the intramolecular

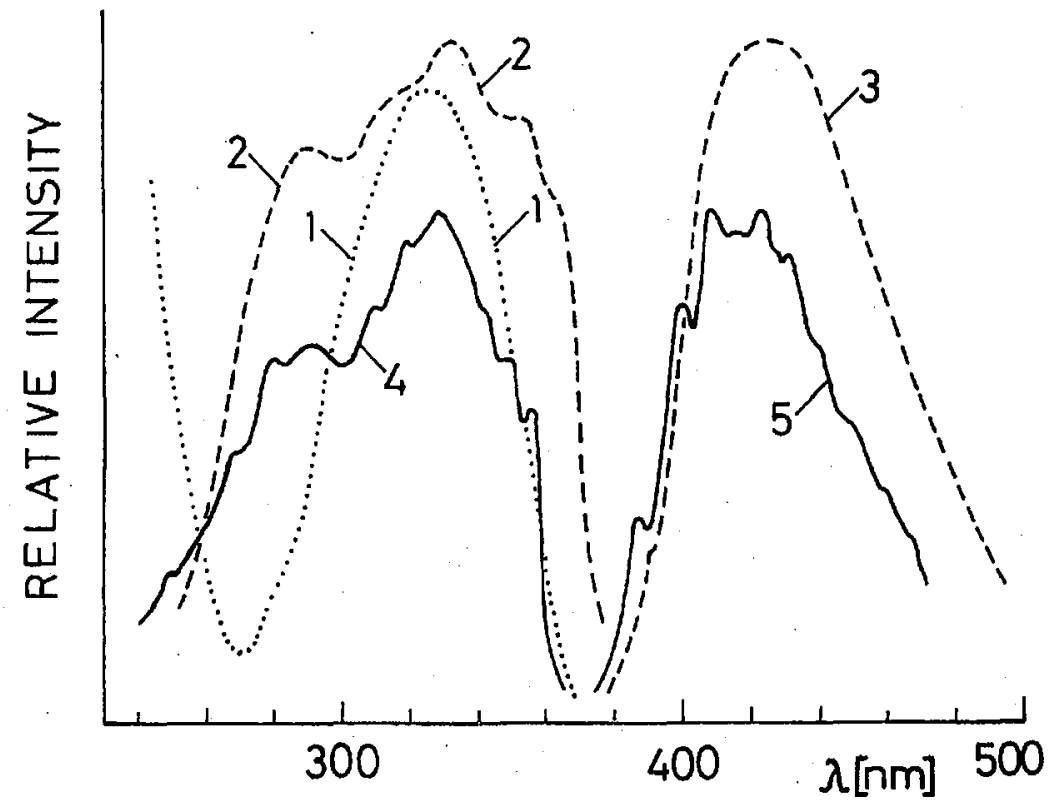

Fig. 4. Electronic spectra of polycrystalline salicylamide at $293 \mathrm{~K}(1,2$ and 3$)$ and $77 \mathrm{~K}(4$ and 5 ): 1 - absorption (function $F$ ); 3 and 5 - fluorescence emission; 2 and 4 - fluorescence excitation. Spectra wére obtained by excitation at $310 \mathrm{~nm}$ and by monitoring at $426 \mathrm{~nm}$. The sample was the mixture of salicylamide powder and $\mathrm{MgO}$ powder in a mass ratio $1: 100$. 
proton-transferred SAM species. The excitation spectrum at room temperature correlates well with the absorption. A similar situation occurs with some analogous compounds (in which the excited state intramolecular proton transfer appears) as 2 -(2'-hydroxyphenyl)benzothiazole and 2-(2'-hydroxy-5'-methylphenyl)benzotriazole when the spectra in crystal and solutions are compared [16]. The excitation spectrum is complex and is observed in the range of $260-370 \mathrm{~nm}$. It is probably composed of two bands with maxima at about 280 and $333 \mathrm{~nm}$. No emission dependence on the excitation wavelength was observed.

The emission spectrum with a maximum at $419 \mathrm{~nm}$, and excitation spectrum with maximum at $329 \mathrm{~nm}$, registered at $77 \mathrm{~K}$, have a vibrational substructure (Fig. 4). The long-wavelength peak of this structure in the excitation spectrum is at about $359 \mathrm{~nm}$ (at room temperature at about $366 \mathrm{~nm}$ ), and we think that this value corresponds to the $0-0$ transition in excitation (absorption). It is in full agreement with the value of $358.7 \mathrm{~nm}$, previously found for SAM as guest molecule in durene mixed crystal [9]. The Stokes shift of SAM fluorescence in polycrystal, which amounts to about $6500 \mathrm{~cm}^{-1}$ and is weakly temperature dependent, is considerably reduced compared to the one in solutions, which is about $10000 \mathrm{~cm}^{-1}$ in hydrocarbon and some aqueous liquid solutions, and about $8000 \mathrm{~cm}^{-1}$ in ethanolic glass solution at $77 \mathrm{~K}$. Also, there is a substantial expansion of the range of the absorption, and especially of the excitation spectrum, on the longwave side towards the region of $340-370 \mathrm{~nm}$. All these facts indicate that the form of the potential surface along the direction of proton transfer at tautomerisation is considerably changed in crystal, in both ground and excited electronic states.

The frequencies of the $\mathrm{N}-\mathrm{H}\left(3413+3460 \mathrm{~cm}^{-1}\right)$ and $\mathrm{O}-\mathrm{H}\left(3200 \mathrm{~cm}^{-1}\right)$ vibrations observed in IR spectrum of crystal powder are considerably different from the corresponding frequencies $\left(3435+3550\right.$ and $\left.2940 \mathrm{~cm}^{-1}\right)$ in solution, which also indicates the difference in potential curves along the $\mathrm{H}$-bond.

\section{Conclusion}

We have studied the absorption, fluorescence emission and fluorescence excitation spectra of polycrystalline salicylamide at room temperature and liquid nitrogen temperature. The fluorescence emission is attributed to the proton-transferred species, arising from excitation of the neutral molecule with intramolecular $\mathrm{H}$-bond in which phenolic group serves as the proton donor to carbonyl group. The excitation spectrum of polycrystalline SAM is considerably widened on the longwave side, compared to that in solution, and its vibrational structure makes possible to determine the 0-0 transition.

The examination of SAM in different solid solutions showed that all three protolytic forms (anion, neutral molecule and cation) exhibit fluorescence, as well as phosphorescence. It seems that phosphorescence is a suitable spectral characteristic for identification of particular molecular forms, because zwitterionic form exhibits no phosphorescence. A most suitable environment for the appearance of neutral molecule (trans-conformer) luminescence is ethanolic solution. Equilibrium constants of the protolytic reactions in excited state were also determined. 


\section{References}

[1] A. Weller, Z. Electrochem. 60, 1144 (1956).

[2] W. Klöpffer, G.J. Naundorf, J. Lumin. 8, 457 (1974).

[3] K. Sandros, Acta Chem. Scand., Ser. A 30, 761 (1976).

[4] A.U. Acuña, J. Catalán, F. Toribo, J. Phys. Chem. 85, 241 (1981).

[5] F. Toribo, J. Catalán, F. Amat, A.U. Acunã, J. Phys. Chem. 87, 817 (1983).

[6] G.J. Woolfe, P.J. Thistlethwaite, J. Am. Chem. Soc. 102, 6917 (1980).

[7] C.M. Estevez, M.A. Rios, J. Rodriguez, Structural Chemistry 3, 381 (1992).

[8] T. Nishiya, S. Yamauchi, N. Hirota, Y. Fujiwara, M. Itoh, J. Am. Chem. Soc. 108, 3880 (1986).

[9] T. Nishiya, S. Yamauchi, N. Hirota, M. Baba, I. Hanazaki, J. Phys. Chem. 90, 5730 (1986).

[10] J. Catalán, J. Palomar, J.L.G. Depaz, J. Phys. Chem. A 101, 7914 (1997).

[11] A.U. Acunã, A. Costelo, J.M. Muñoz, J. Phys. Chem. 90, 2807 (1986).

[12] T.M. Surányi, E.A. Durendić, D.A. Miljković, J. Serb. Chem. Soc. 53, 551 (1988).

[13] I. Gúth, I. Janić, M. Kakaš, E. Durendić, T, Surányi, J. Mol. Struct. 266, 367 (1992).

[14] A.C. Marfunin, Vvedenie v fiziku minerallov, Nedra, Moskva 1974.

[15] A. Agren, Svensk Kem. Tidskr. 68, 189 (1956).

[16] M. Wiechmann, H. Port, J. Lumin. 48\&49, 217 (1991). 